\title{
Die Bedeutung von Infinitiv und Konjunktiv als Modi der Nicht-Mitteilung (unter besonderer Berücksichtigung des Italienischen)
}

\author{
Claudia Pichler (Klagenfurt)
}

\begin{abstract}
The infinitive (also referred to as "Nennform") is positioned both formally and functionally between noun and verb. This so-called "intermediate status" and the pragmaticcommunicative characteristics/functions resulting from it are described on the basis of a selection of Old Italian language samples. The study therefore examines the correlation between morphosyntactic and informational structure and focuses in particular on the morphosyntactic codification of thematic elements of expression. An accordance can be ascertained regarding the pragmatic-communicative characteristics/functions of the infinitive (i.e. infinitive constructions of the type: definite article + infinitive + subject) and the (thematic) subjunctive.
\end{abstract}

\section{$1 \quad$ Einleitung}

Neben Indikativ und Konjunktiv wird mitunter auch der Infinitiv zu den Modi des Verbs gezählt. Im Folgenden soll geklärt werden, durch welche gemeinsamen pragmatischkommunikativen Eigenschaften bzw. Funktionen Infinitiv und Konjunktiv gekennzeichnet sind und inwieweit es gerechtfertigt ist, den Infinitiv als Modus zu bezeichnen. Im Mittelpunkt stehen dabei insbesondere durch einen Artikel eingeleitete, syntaktisch abhängige Infinitivphrasen, ihre morphosyntaktische Struktur sowie ihr jeweiliger Informationsstatus. Die Beispielsätze stammen größtenteils aus altitalienischen Texten, da gerade die "ältere" Sprache zahlreiche und verschiedenartige Infinitivkonstruktionen aufweist, die für die vorliegende Untersuchung von Interesse sind.

\section{Exkurs: Nominale und verbale Merkmale des Infinitivs/ Der Infinitiv - ein Modus?}

Unter einem verbum infinitum versteht man im Allgemeinen eine unkonjugierte, das heißt hinsichtlich Person, Numerus, Tempus und Modus nicht bestimmte Verbform. Als sogenannte Nominalform des Verbs (auch Nennform genannt) steht der Infinitiv formal und funktional zwischen Verb und Nomen. Cf. dazu Mayerthaler/Fliedl/Winkler (1993: 134-135):

Besonders deutlich zeigt sich die Doppelnatur von [...] Infinitivgruppen im Italienischen. Hier finden sich Infinitivstrukturen mit Artikel, deren syntaktische Merkmale sowohl auf den nominalen als auch auf den verbalen Charakter dieser markierten Konstruktionen hindeuten [...].

Man betrachte in diesem Zusammenhang die drei folgenden Beispiele:

a) Ma egli si vorrebbe dare per legge ad ognuno; a' volgari il non parlare latinamente, [...]; a' litterati, che mai da loro [...] non si parlasse volgare alla maniera degli ignoranti: ... (Dialogo delle lingue: 168) 
(Allen müsste man daher folgendes zum Gesetz machen : dem Volk, nicht lateinisch zu sprechen, [...], den Gebildeten, niemals die Volkssprache zu gebrauchen wie die Unwissenden, ...)

b) in modo che tu hai inimici tutti quelli che hai offesi in occupare quello principato, e non ti puoi mantenere amici quelli che vi ti hanno messo, per non li potere satisfare in quel modo che si erano presupposto e per non potere tu usare contro a di loro medicine forti, sendo loro obligato ; (Il Principe, Kap. III: 12)

(Damit machst du dir alle zu Feinden, die du bei der Besetzung jenes Herrschaftsgebiets geschädigt hast, und du kannst dir nicht die Freundschaft derer bewahren, die dich in ihr Land geholt haben, weil du sie nicht ihren Erwartungen gemäß zufrieden stellen und weil du ihnen gegenüber keine harten Maßnahmen ergreifen kannst, da du ihnen verpflichtet bist.)

c) ...per essere el sangue de' loro antiqui signori spento, ... (Il Principe, Kap. IV: 34)

(da nämlich das Geschlecht ihrer ehemaligen Herren ausgetilgt war, ...)

Die Infinitivphrasen in a) - c) sind sowohl durch nominale als auch durch verbale Eigenschaften gekennzeichnet. Als ein typisch nominales Merkmal der Infinitivphrase ist neben dem infiniten verbalen Kern - deren Verbindung mit einem Artikel bzw. mit einer Präposition zu nennen (il non parlare .../per non potere ...).

Als Indikatoren für die Verbalität der Infinitivkonstruktion gelten ${ }^{1}$ :

1. das jeweils nominativische Subjekt (per non potere tu .../per essere el sangue ...),

2. die Passivdiathese (essere ... spento $\rightarrow$ "Zustandspassiv"),

3. die Negationspartikel (il non parlare .../per non (li) potere ...),

4. die Verbindung Modalverb + Infinitiv (non li potere satisfare/non potere tu usare...),

5. die Verbindung des Infinitivs mit einem Adverb (parlare latinamente),

6. die von der Valenz des Infinitivs geforderten Komplemente (li potere satisfare/non potere tu usare ... medicine forti).

Der verbale Charakter des Infinitivs ist vor allem auch dann offensichtlich, wenn die Verbalphrasen unabhängiger, nicht-subordinierter Sätze anstelle eines finiten Verbums eine Infinitiv-Form aufweisen. Das heißt, das Zustandekommen einer gültigen Prädikation ist nicht notwendigerweise an das Vorhandensein einer finiten Verbform gebunden, wie die folgenden Beispiele $^{2}$ zeigen:

Verneinter Imperativ: Non fare così! (Mach es nicht auf diese Weise!) [übers. v. Verf.]

"Frasi nominali" des Typs: Dove andare ? (Wohin sollen wir gehen?) [übers. v. Verf.] Che dirgli? (Was sollen wir ihm sagen?) [übers. v. Verf.]

Historischer (narrativer) Infinitiv: E noi a ridere. (Und wir haben gelacht.) [übers. v. Verf.]

Ob nun Äußerungen, die als verbalen Nukleus eine infinite Verbform enthalten, als Sätze zu klassifizieren sind oder nicht, ist eine Frage der Definition. Wichtiger ist die Tatsache, dass in den soeben genannten Beispielen jeweils eine Prädikation erfolgt und das kommunikative Ziel des Sprechers erreicht wird. Cfr. Ramat (2002: 412):

... occorre $[\ldots .$.$] tener presente anche l'aspetto pragmatico, cioè gli usi concreti in cui forma$ grammaticale infinitiva compare. Esistono infatti frasi infinitivali con particolari valori modali [Hvhbg. v. Verfass.] e semanticamente autosufficienti [...].

\footnotetext{
${ }^{1}$ Cfr. Mayerthaler/Fliedl/Winkler, Infinitivprominenz in europäischen Sprachen, 5.3.5.4. "Zur Doppelnatur einiger Infinitivkonstruktionen im Italienischen und Altfranzösischen": 134-139.

2 Die Beispiele stammen aus Jansen/Polito/Strudsholm, "Dialogo vago sull'infinito e altro": 13.
} 
(es ist nötig, auch den pragmatischen Aspekt zu berücksichtigen, das heißt die konkreten Anwendungen, in denen die grammatikalische Form des Infinitivs erscheint. Es gibt in der Tat infinitivhaltige Sätze, die bestimmte modale Merkmale aufweisen und die semantisch unabhängig sind.) ${ }^{3}$ [übers. v. Verf.]

Im Hinblick darauf, dass manche Sprachen (z. B. das Portugiesische) flektierte personale Infinitivformen aufweisen, wird deutlich, dass es zwischen finiter Verbform einerseits und infinitem Verbum andererseits noch Zwischenstufen gibt - ein "continuum con forme più o meno finite" (Jansen/Polito/Strudsholm 2002: 14) (ein Kontinuum mit mehr oder weniger finiten Formen). Die Zuordnung des Infinitivs zu einer morphologischen Kategorie (im Sinne einer durch spezifische grammatische Aspekte gekennzeichneten Wortart) erweist sich aufgrund seines Zwischenstatus zwischen Nomen und Verb als problematisch. Ramats Definition (Ramat 2002: 413) erscheint daher angebracht, wonach der Infinitiv nicht als morphologische Kategorie, sondern vielmehr als ein Wert ("un valore") der verbalen Eigenschaft MODUS betrachtet wird ("un 'valore' della 'caratteristica' MODO della categoria VERBO: VB (verbo) $\rightarrow$ MD (modo) $\rightarrow$ INF (infinito)").

Man betrachte in diesem Zusammenhang auch den sogenannten modalen Infinitiv im Russischen, der sich aus "dem potentiellen Agens, das im Dativ ${ }^{4}$ genannt wird, und einem unabhängigen Infinitiv zusammensetzt" (Maurice 1996: 72). Nach Maurice (1996: 14) ist der modale Infinitiv, der neben Fragesätzen auch in Aussagesätzen vorkommt, "ein syntaktisches Mittel zum Ausdruck von Modalität. Die Modalität ist hierbei implizit und durch die Konstruktion bedingt". Was die pragmatisch-kommunikative Verwendung des modalen Infinitivs betrifft, so ist anzumerken, "dass Sätze mit modalem Infinitiv meist Hintergrundinformation oder Erklärungen zu irgendwelchen Handlungen oder Unterlassungen bereits eingeführter Personen geben [...]" (Maurice 1996: 73).

Im vorliegenden Beitrag wird der (durch einen Artikel eingeleitete) Infinitiv insofern als Modus $^{5}$ betrachtet, als er

1. ein morphosyntaktisches Mittel zur Kennzeichnung jener Äußerungselemente darstellt, die einen bestimmten Inhalt nicht behaupten, sondern präsupponieren und folglich als thematisch ausweisen $(\rightarrow$ in diesem Sinne fungiert der Infinitiv - ebenso wie der thematische Konjunktiv - gewissermaßen als "Modus der Nicht-Mitteilung" (Gsell/Wandruszka 1986: 66)); und

2. als typischer Unterordnungsmodus hauptsächlich in abhängigen Sätzen vorkommt.

\section{Strukturelle, inhaltliche und pragmatisch-kommunikative Relationen}

Zwischen einzelnen Sätzen bzw. Satzteilen (Phrasen) bestehen Relationen unterschiedlicher Art, die nach Vallauri wie folgt klassifiziert werden können (cfr. Vallauri 1996: 13-14):

- $\quad$ syntaktische Relationen; diese betreffen den Grad der syntaktischen Abhängigkeit (in Anlehnung an Halliday (1985: 193-195) als interdipendenza bezeichnet: Nebenordnung, Unterordnung, Einbettung);

- Relationen, die durch Koreferenz entstehen;

- durch Projektion bedingte Relationen (sog. "relazioni di proiezione") (Vallauri 1996: 14), die sich dadurch ergeben, dass der Sprecher die Äußerung eines bestimmten

\footnotetext{
${ }^{3}$ Cfr. Skytte, La sintassi dell'infinito in italiano moderno: 16.

${ }^{4}$ Hinsichtlich der Subjektseigenschaften des Nomens im Dativ cfr. Franks 1995: 253-256.

${ }^{5} \mathrm{Cfr}$. dazu auch Winkler, Modus und Modalität im Italienischen am Beispiel von Infinitiv und Konjunktiv.
} 
Inhalts nicht $\mathrm{zu}$ seiner eigenen Behauptung macht, sondern diesen Inhalt lediglich übernimmt bzw. wiedergibt (cfr. indirekte Rede, Zitat);

- logisch-semantische Relationen/Sinnrelationen, sowie

- Relationen, welche die Informationsstuktur der Äußerung betreffen ("relazioni di informatività"); diese umfassen pragmatisch-kommunikative Kategorien wie:

- Vordergrund (primo piano)/Hintergrund (sfondo),

- präsupponiert (presupposto)/behauptet (asserto),

- Thema/Rhema,

- alte (dato) und neue (nuovo) Information etc.

Der Informationsstatus einer Konstituente (d. h. thematisch od. rhematisch, assertierend, nicht-assertierend etc.) wird durch unterschiedliche sprachliche Mittel gekennzeichnet. Dazu zählen u. a.:

- der Modus des Verbs (cfr. thematischer Konjunktiv),

- die Wort- und Satzgliedstellung,

- der Grad der syntaktischen Abhängigkeit,

- die Verwendung bestimmter Konnektiva, sowie

- die Intonation.

Die morphosyntaktische Struktur einer Äußerung ist demnach Ausdruck ihrer spezifischen Informationsstruktur. Vallauri (1996: II-III) spricht in diesem Zusammenhang von "sintassi strutturale" (die syntaktisch-strukturelle Ebene der Äußerung betreffend) und von "sintassi dell'informazione" (die Informationsstruktur der Äußerung betreffend) - zwei sich wechselseitig beeinflussende Ebenen.

Im vorliegenden Beitrag gilt es, diese Wechselbeziehung zwischen morphosyntaktischer Struktur und Informationsstruktur zu beschreiben bzw. aufzuzeigen, wie der Informationsstatus einer Konstituente durch morphosyntaktische Mittel zum Ausdruck gebracht wird. Im Zentrum steht dabei vor allem die morphosyntaktische Kodierung thematischer Äußerungselemente, wobei hinsichtlich der pragmatisch-kommunikativen Funktionen von Infinitiv und Konjunktiv bestimmte Übereinstimmungen festzustellen sind.

\section{$4 \quad$ Assertion - Projektion - Präsupposition}

Man betrachte vorab die zwei folgenden Beispiele:

(1) Mi piace molto/è bello che Paolo sia arrivato ieri sera.

(Es gefällt mir sehr/es ist schön, dass Paolo gestern Abend angekommen ist.)

(2) ... mi piace noi essere entrati a dimostrare con le novelle quanta sia la forza delle belle e pronte risposte, ... (Decameron, 1. Tag, 5. Novelle: 68)

(Es gefällt mir, dass wir dahin gekommen sind, mit unseren Novellen zu zeigen, wie groß die Wirkung anmutiger und schlagfertiger Antworten ist.)

Das primäre Mitteilungsziel des Sprechers besteht in (1) nicht in der Feststellung, dass Paolo angekommen ist, sondern vielmehr im Ausdruck der Freude über diesen Umstand.

Oder anders formuliert:

Der Inhalt des untergeordneten Satzes wird vom Sprecher nicht behauptet (im Sinne von:

"Paolo è arrivato ieri sera"), sondern präsupponiert. Das heißt,

[...] tali contenuti sono posti su un livello diverso da quello su cui il producente esercita la sua funzione referenziale. (Vallauri 1996: 36) 
(... derartige Inhalte werden auf eine andere Ebene gestellt als jene, auf die der Sprecher seine referentielle Funktion ausübt.) [übers. v. Verf.]

Dasselbe gilt auch für Beispiel (2), das anstelle eines konjunktivischen Komplementsatzes eine Infinitivphrase enthält, deren Inhalt vom Sprecher als existentiell gegeben vorausgesetzt wird:

Mi piace noi essere entrati ...

Mi piace il fatto che noi siamo entrati ...

Die Äußerung eines bestimmten Inhalts (Proposition) kann demnach auf unterschiedlichen Ebenen erfolgen, und zwar abhängig davon, inwieweit der Sprecher diesen Inhalt

a) selbst behauptet (illokutionärer Akt: Assertion),

b) übernimmt bzw. wiedergibt (z. B. Zitat, indirekte Rede $\rightarrow$ nach Vallauri (1996: 34-35) als "proiezione di locuzioni e idee" bezeichnet), oder

c) präsupponiert (= "proiezione di fatti" - s.u.).

Es handelt sich somit um unterschiedliche Präsentationsebenen ein- und desselben Inhalts, die durch spezifische morphosyntaktische Mittel gekennzeichnet sind. Um beispielsweise einen Äußerungsteil als projiziert auszuweisen, verwendet das Deutsche in der indirekten Rede generell den Konjunktiv (cfr. im Gegensatz dazu: Assertion $\rightarrow$ Indikativ).

Neben der "proiezione di locuzioni e idee" (Zitat, indirekte Rede) verweist Vallauri (1996: 36) (in Anlehnung an Halliday) noch auf einen weiteren Projektionstyp, der sogenannten "proiezione di fatti". Dabei wird ein bestimmter Inhalt nicht behauptet (weder vom Sprecher selbst, noch von einer anderen Person $\rightarrow$ cfr. indirekte Rede), sondern präsupponiert und folglich durch entsprechende nicht-assertorische Äußerungselemente kodiert (cfr. konjunktivischer Komplementsatz in (1), Infinitivkonstruktion in (2)).

Als implizite Sinnvoraussetzungen stellen präsupponierte Sachverhalte zumeist bekannte, also im Bewusstsein der Gesprächsteilnehmer bereits verankerte und somit thematische Informationselemente dar. Diesem Faktum wird gerade durch die Verwendung des thematischen Konjunktivs (s. dazu weiter unten) Rechnung getragen. In (1) dient der Konjunktiv also nicht nur als Zeichen syntaktischer Unterordnung, sondern insbesondere auch als Kennzeichen des Mitteilungswertes (thematisch $\rightarrow$ bekannte Information) und der Präsentationsebene des Nebensatzinhalts. Letzterer wird vom Sprecher nicht behauptet (cfr. Assertion $\rightarrow$ Indikativ als Modus der Mitteilung), sondern als präsupponierter Sachverhalt sozusagen indirekt zum Ausdruck gebracht (s. o. "proiezione di fatti"). In diesem Sinne fungiert der thematische Konjunktiv gewissermaßen als "Modus der Nicht-Mitteilung" (siehe unten).

Bevor nun Infinitiv und Konjunktiv im Hinblick auf ihre gemeinsamen kommunikationsspezifischen Funktions- und Verwendungsweisen untersucht werden, soll im gleich anschließenden Kapitel noch einmal in aller Kürze die Theorie der Funktionalen Satzperspektive erläutert werden.

\section{Theorie der Funktionalen Satzperspektive}

Die Theorie der Funktionalen Satzperspektive (auch Thema-Rhema-Gliederung genannt) beschäftigt sich "mit dem Mitteilungswert sprachlicher Einheiten und den Auswirkungen des Mitteilungswertes auf den Aufbau sprachlicher Äußerungen" (Tschida 1995: 1). Der Begriff des Mitteilungswertes ist in der Linguistik nicht eindeutig definiert: "Üblicherweise wird zwischen thematischem (= geringem) und rhematischem (= hohem) Mitteilungswert unterschieden, wobei das Thema dem bekannten oder als bekannt unterstellten Teil des Satzinhalts entspricht und das Rhema der neuen Information" (Blumenthal 1987: 36). 
Mitunter wird das Thema auch als Satzgegenstand (= das, worüber man spricht) und das Rhema als Satzaussage (= das, was über das Thema ausgesagt wird) verstanden. In diesem Sinne ist das Rhema in erster Linie für die Textprogression und das Thema für die Textkontinuität verantwortlich. Dass diese Definition (Thema - Satzgegenstand, Rhema Satzaussage) nicht unproblematisch ist, zeigt das folgende Beispiel:

(3) If the duke gives anything to my aunt, it'll be that teapot (Vallauri 1996: 39)

(Falls der Herzog meiner Tante irgendetwas gibt, so wird es diese Teekanne sein.) [übers. v. Verf.]

In (3) ist nicht the duke das Thema, sondern der an der ersten Stelle des Satzgefüges befindliche Konditionalsatz. Der darauf folgende Hauptsatz ist rhematisch ${ }^{6}[6]$. Um also die Informationsverteilung innerhalb eines Satzgefüges $\mathrm{zu}$ bestimmen, müssen Thema und Rhema nach anderen Kriterien definiert werden. Vallauri spricht für eine Definition, die auf dem Konzept des sogenannten "Mitteilungsziels" basiert, wonach sich das Thema tendenziell mit den sogenannten "clausole di tipo 'circostanziale'" - also mit dem abhängigen Satz - und das Rhema mit dem Hauptsatz identifizieren lässt. Der Hauptsatz ist - im Vergleich zum Nebensatz - "portatrice di una più forte illocutività" (Vallauri 1996: 42), das heißt der eigentliche Träger der illokutionären Kraft. Cfr. (Vallauri 1996: 40-41):

[...] ci conviene far uso del concetto di scopo dell'atto comunicativo, applicandolo al complesso clausale. [...] in molti complessi clausali è possibile individuare una gerarchia di importanza delle clausole che li compongono, secondo il loro codificare o meno il contenuto che costituisce lo scopo del messaggio [...]. [...] La prima parte in sostanza fornisce un corredo di elementi necessari a poter comprendere la seconda, la quale [...] codifica $[\ldots][\ldots]$ la parte di messaggio che è portatrice della forza illocutiva.

(... es ist angebracht, vom Konzept des Mitteilungsziels Gebrauch zu machen und es auf das Satzgefüge anzuwenden. [...] In vielen Satzgefügen kann eine Hierarchie hinsichtlich der Wichtigkeit der einzelnen Teilsätze festgestellt werden, und zwar in Abhängigkeit davon, inwieweit die Teilsätze den Inhalt kodieren, der das Mitteilungsziel der Äußerung darstellt. [...] Der erste Teil [des Satzgefüges] liefert im Wesentlichen jene Elemente, die nötig sind, um den zweiten Teil der Äußerung verstehen zu können, der Träger der illokutionären Kraft ist.) [übers. v. Verf.]

Auch im Folgenden beziehen sich die Begriffe Thema/thematisch und Rhema/rhematisch auf den vom jeweiligen Mitteilungsziel abhängigen Mitteilungswert sprachlicher Äußerungen. Diese auf dem Konzept des Mitteilungsziels beruhende Definition von Thema und Rhema erweist sich für die vorliegende Untersuchung als angebracht, gilt es doch, die (gemeinsamen) pragmatisch-kommunikativen Funktionen von Infinitiv und Konjunktiv aufzuzeigen, die typischerweise in abhängigen Sätzen bzw. Phrasen vorkommen (cfr. Konjunktiv als Nebensatzmodus, Nebensatzverkürzung durch infinites Verbum).

\footnotetext{
${ }^{6}$ Die generelle Abfolge "Thema vor Rhema" lässt sich nicht nur im einfachen Satz, sondern auch innerhalb des Satzgefüges beobachten.
} 
Man betrachte in diesem Zusammenhang (4)-(9): Die Thematizität (sprich der geringere Mitteilungswert) des jeweils untergeordneten Satzes ist gleich zweifach markiert 7 :

1. durch seine Stellung innerhalb des Satzgefüges (der Nebensatz geht dem Hauptsatz voran);

2. durch die infinite Verbform.

(4) E così dicendo e avendo del tutto mutato proposito da quello per che andato v'era, fattosi più presso alla giovane, pianamente la cominciò a confortare e a pregarla che non piagnesse; (Decameron, 1. Tag, 4. Novelle: 64)

(Nachdem er sich das überlegt und so den Vorsatz, um dessentwillen er gekommen war, ganz und gar umgestoßen hatte, ging er auf das Mädchen zu und begann, sie freundlich zu trösten und zu bitten, nicht länger zu weinen;)

(5) Fattasi adunque la via insegnare, non trovando alcun che v'andasse, temette non per isciagura gli venisse smarrita e quinci potere andare in parte dove così tosto non troveria da mangiare; (Decameron, 1. Tag, 7. Novelle: 84)

(Nachdem er sich den Weg hatte beschreiben lassen, aber niemanden fand, der auch dorthin ging, fürchtete er, dass er sich unglücklicherweise verirren und so irgendwohin gelangen könnte, wo er so bald nichts zu essen fände;)

(6) E appresso mangiare [...] il fé nobilmente vestire, ... (Decameron, 1. Tag, 7. Novelle: 88)

(So ließ er ihn nach dem Essen [...] vornehm einkleiden, ...)

(7) e quelle, per essere el sangue de' loro antiqui signori spento, non riconoscevano se non è Romani. (Il Principe, Kap. IV: 34)

(da nämlich das Geschlecht ihrer ehemaligen Herren ausgetilgt war, erkannten diese Provinzen allein die Römer als Herren an.)

(8) Onde è da notare che, nel pigliare uno stato, debbe l'occupatore di esso discorrere tutte quelle offese che gli è necessario fare; (Il Principe, Kap. VIII: 72)

(Demnach ist festzuhalten, dass bei der Aneignung eines Staates der Eroberer alle Gewalttaten in Betracht ziehen muss, die zu begehen nötig ist, ...)

(9) Francesco Sforza, per essere armato, di privato diventò duca di Milano; (Il Principe, Kap. XIV: 112)

(Francesco Sforza stieg vom Privatmann zum Herzog von Mailand auf, weil er gerüstet war;)

Ändert sich die Abfolge der einzelnen Teilsätze innerhalb des Satzgefüges, so ändert sich auch dessen Informationsstruktur. Man betrachte (10) - (11):

Nicht der Hauptsatz, sondern die abhängige Infinitivphrase stellt das Informationszentrum des Satzes dar. Diese wird dem Hauptsatz nachgestellt und dadurch als rhematisch gekennzeichnet. Der Infinitiv kennzeichnet die Phrase als nicht-assertierend, das heißt, der Inhalt der Infinitivphrase wird nicht behauptet, sondern präsupponiert (cfr. Assertion vs. Präsupposition):

(10) Ha perduto, adunque, il re Luigi la Lombardia per non avere osservato alcuno di quelli termini osservati da altri che hanno preso provincie e volutole tenere. (Il Principe, Kap. III: 28)

(Somit hat König Ludwig die Lombardei verloren, weil er keine jener Regeln beachtet hat, die von anderen beachtet wurden, die Provinzen erobert hatten und behalten wollten.)

\footnotetext{
${ }^{7}$ Inwieweit man Phrasen, die keine finite Verbform enthalten, als Nebensätze bezeichnen kann, ist eine Frage der Definition. Da solche Phrasen jedoch die syntaktische Funktion eines Nebensatzes erfüllen, sollen sie auch weiterhin unter diesem Terminus subsumiert werden. Infinitivhaltige Phrasen werden im Folgenden auch als "Infinitivphrasen" bezeichnet.
} 
(11) Le cagioni della difficultà in potere occupare il regno del Turco sono per non potere essere chiamato da' principi di quello regno, né sperare, con la rebellione di quelli ch'egli ha d'intorno, potere facilitare la sua impresa. (Il Principe, Kap. IV: 32)

(Die Gründe für die Schwierigkeiten, die es macht, das Reich des Türken zu erobern, bestehen darin, dass man weder von den Würdenträgern dieses Reiches herbeigerufen werden kann noch hoffen darf, durch einen Aufstand derer, die den Herrscher umgeben, sich die Eroberung zu erleichtern.)

\section{Infinitiv und Konjunktiv als Modi der "Nicht-Mitteilung"}

Der neutrale Inhalt/Sachverhalt eines Satzes wird bekanntlich als Proposition bezeichnet. Erst durch verschiedene lexikalische Mittel (Satzadverbien, Modalverben), vor allem aber durch die verbale Kategorie des Modus wird - neben der inhaltlichen Formulierung der Äußerung auch die subjektive Einstellung des Sprechers zum Satzinhalt ausgedrückt. Während der Indikativ gerade durch seine assertierend-mitteilende Funktion bestimmt ist (d.h. einen Sachverhalt als real darstellt) und folglich auch als "Wirklichkeitsform" oder als "Normalform" bezeichnet wird, erweist sich die Festlegung des Konjunktivs auf eine einheitliche Grundfunktion als problematisch (s. dazu auch Kapitel 6).

Im klassischen Latein erfüllte der Konjunktiv unterschiedlichste Funktionen. Neben der volitiven Funktion ist insbesondere auch auf die dubitative Funktion im Bereich der epistemischen Modalität (= "Einschätzung der Gültigkeit einer Aussage beziehungsweise des Bestehens eines Sachverhaltes durch den Sprecher" (Gsell/Wandruszka 1986: 46)) hinzuweisen:

Die Tatsache, dass man es hier mit den verschiedensten Graden und Arten des Vorbehaltes gegenüber der Wahrheit des Nebensatzes zu tun hat, lässt bereits erkennen, dass sich auch der Modusgebrauch weniger einheitlich darstellt und stärkeren Schwankungen unterworfen ist [...]. (Gsell/Wandruszka 1986: 46f.)

Man betrachte in diesem Zusammenhang die folgenden Beispiele:

(12) Ital.:

Non credo che Maria abbia superato l'esame. (Konjunktiv)

(13) Frz.: Je ne crois pas que Marie ait réussi à l'examen. (Konjunktiv)

(Ich glaube nicht, dass Maria die Prüfung bestanden hat.)

In (12)/(13) fungiert der Konjunktiv nicht nur als Zeichen der syntaktisch-funktionalen Abhängigkeit des Komplementsatzes, sondern er enthält zusätzlich auch eine modale Bedeutungskomponente, nämlich die Nicht-Annahme der Realisierung des Nebensatzinhalts seitens des Sprechers. Gegenüber der Verwendung finiter Verben in Nebensätzen hat die Verwendung infiniter Verbformen den Vorteil, dass infinite Formen prinzipiell modusneutral sind. Im klassischen Latein steht nach den verba sentiendi und dicendi fast ausschließlich der accusativus cum infinitivo:

Während diese Verben (verba sentiendi et dicendi) im klassischen Latein meist mit AcI konstruiert wurden, richtet sich in den entsprechenden spätlateinischen Konjunktionalsätzen die Wahl des Modus nach der jeweiligen Einschätzung ihres Realitätsgehaltes. So steht etwa nach scire, credere vorwiegend der Indikativ, und nach dicere, referre eher der Konjunktiv, und ebenso nach verneintem Hauptsatz wie noch im Neufranzösischen. Diese Modussetzung bleibt im Prinzip bis ins 17. Jh. erhalten. (Gsell/Wandruszka 1986: 65f.)

Im Hinblick auf die spezifisch dubitative Funktion des Konjunktivs lassen sich im Französischen und im Italienischen unterschiedliche Entwicklungstendenzen feststellen. Cfr.:

(14) Ital.: $\quad$ Credo che Maria abbia superato l'esame. (Konjunktiv)

(15) Frz.: Je crois que Marie a réussi à l'examen. (Indikativ) 
(Ich glaube, dass Maria die Prüfung bestanden hat.)

Während im Französischen das von positiven Verben des Glaubens und Meinens abhängige Prädikat im Indikativ erscheint (15), fordert das Italienische auch in diesem Kontext den Konjunktiv (14). Nach verneinten Verben des Glaubens und Meinens steht in beiden Sprachen jeweils der Konjunktiv (12, 13). Ähnliche Verhältnisse finden sich auch in Nebensätzen, die von einem deklarativen Verbum im Hauptsatz abhängen: Indikativ prinzipiell nach positivem Hauptsatz, Konjunktiv mitunter nach Verneinung und Frage, "zumal wenn der Sprecher Zweifel an der Wahrheit des Nebensatzes anklingen lassen will" (Gsell/Wandruszka 1986: 67). Cf. dazu das folgende Beispiel ${ }^{8}$ :

(16) Non ho mai sostenuto che gli altri non ne fossero capaci. (Konjunktiv)

(Ich habe niemals behauptet, dass die anderen nicht dazu fähig seien.)

Zusammenfassend kann festgestellt werden, dass

das Italienische die charakteristische Entwicklung des Französischen seit dem 17. Jh. nicht vollzogen hat. Der Konjunktiv wird hier nach den Verben des Glaubens und Meinens nicht nur im Falle des Zweifels oder der Negierung der Wahrheit des Nebensatzes gesetzt, sondern er dient generell auch zur Nuancierung des Wahrheitsanspruches einer positiv formulierten Annahme. Wir können nach solchen Verben sogar eine ausgeprägte Tendenz zum Congiuntivo feststellen. (Gsell/Wandruszka 1986: 66)

Neben dem volitiven (od. deontischen) und dem dubitativen (od. epistemischen) Konjunktiv breitet sich im Französischen im Laufe des 16. Jh. ein weiterer Typus aus, der sogenannte thematische oder faktive Konjunktiv. Wie schon der Name sagt, besteht die Funktion des Konjunktivs in diesem Falle darin, die Thematizität sprachlicher Elemente zu kennzeichnen. Der thematische Konjunktiv findet sich insbesondere in Subjektsätzen und in proleptischen Komplementsätzen, sowie nach Verben und Ausdrücken des Affekts bzw. der subjektiven Beurteilung 9:

Frz.:

(17) Qu'elle soit pauvre, c'est certain.

(Dass sie arm ist, (das) ist sicher.)

(Aber: Il est certain qu'elle est pauvre.)

(18) Qu'elle soit pauvre, je le crois.

(Dass sie arm ist, (das) glaube ich.)

(19) C'est dommage/je suis heureux/je regrette qu'il soit parti.

(Es ist schade/ich bin glücklich/ich bedaure, dass er abgefahren ist.)

Ital.:

(20) $\grave{E}$ logico che gli scienziati cerchino/*cercano di risolvere questo problema con la massima urgenza. (Konjunktivverwendung in gehobenem Register) ${ }^{10}$

(Es ist naheliegend, dass die Wissenschaftler versuchen, dieses Problem umgehend zu lösen.) [übers. v. Verf.]

\footnotetext{
${ }^{8}$ Zitiert aus: Reumuth/Winkelmann, Praktische Grammatik der italienischen Sprache: 220.

${ }^{9}$ Die Beispiele (17)-(19) stammen aus: Wandruszka, "Nochmals zum 'thematischen' Konjunktiv": 343.

${ }^{10}$ Zitiert aus: Renzi/Salvi, Grande grammatica italiana di consultazione, Volume II: 476.
} 
(21) È una vergogna che qualcuno di voi non pensi/*pensa ad aiutarci. ${ }^{11}$

(Es ist eine Schande, dass es keinem von euch in den Sinn kommt, uns zu helfen.) [übers. v. Verf.]

Der quasi entmodalisierte thematische Konjunktiv (die Modalität wird in den genannten Beispielen gerade durch den übergeordneten Satz zum Ausdruck gebracht) ist nicht nur ein Indiz syntaktischer Unterordnung, sondern er fungiert als sogenannter "Modus der NichtMitteilung" (cfr. Gsell/Wandruszka 1986: 66, 71f.):

In einer Äußerung wie

Je (ne) regrette (pas) qu'elle soit déjà sortie. (thematischer Konjunktiv)

(Ich bedaure (nicht), dass sie schon ausgegangen ist.)

soll primär nicht kommuniziert werden, dass sie ausgegangen ist, sondern vielmehr das Bedauern des Sprechers darüber. Im Mitteilungszentrum steht die durch den Hauptsatz zum Ausdruck gebrachte persönliche Bewertung, die den fokussierten (rhematischen) Teil der Äußerung darstellt, wobei die Wahrheit des Nebensatzes vom Sprecher präsupponiert wird. Cf.:

Der Voraussetzung der existenziellen Gegebenheit des Sachverhalts entspricht nun auf der kommunikativen Ebene die Voraussetzung des informationellen Gegebenseins. [...] [...] Die Tatsache, dass in solchen Nebensätzen fast ausschließlich der Konjunktiv auftritt, entspricht nun wieder exakt unserer Hypothese über den Grundwert des Subjonctif, nach der dieser Modus im Prinzip die Funktion hat, einen Satz oder Satzteil als Nicht-Mitteilung zu kennzeichnen. Die inhärente Thematizität dieser Nebensätze bedeutet gleichzeitig einen hohen Grad an kommunikativer Unselbständigkeit und Unterordnung [...]. (Gsell/Wandruszka 1986: 72)

Man betrachte in diesem Zusammenhang die folgenden altitalienischen Beispiele.

Die thematischen Äußerungselemente sind in eine Infinitivphrase eingebettet, die durch einen definiten Artikel eingeleitet wird und ein explizit genanntes, dem Infinitiv nachgestelltes (logisches) Subjekt enthält (in (22) und (23) in Form eines klitischen Pronomens). Die Infinitivkonstruktion des Typs "Definiter Artikel + Infinitiv + Subjekt" weist also hinsichtlich ihrer Funktions- und Verwendungsweise Gemeinsamkeiten mit dem thematischen Konjunktiv auf, das heißt, sie fungiert gewissermaßen als "Modus der Nicht-Mitteilung" (cfr. Wunderlis Modushierarchie, Wunderli 1976: 11-27). Cf.:

(22) Onde egli è strana e bella cosa il vederci continuamente vivere e parlare con barbari, $e$ non aver punto del barbaro. (Dialogo delle lingue: 167)

(Denn es ist eine schöne und bemerkenswerte Sache, dass wir fortwährend mit Barbaren leben und sprechen und doch nichts vom Barbarentum annehmen.)

(23) Ma pure stimo l'avermi affaticato apresso di voi non poco mi gioverà, ... (I libri della famiglia, Prologo: 13)

(Und doch, meine ich, wird mein mühevolles Werk mir bei euch nicht wenig nützen;)

(24) Perché questi principi, o comandano per loro medesimi, o per mezzo de' magistrati; nell'ultimo caso, è piú debole e piú periculoso lo stare loro, perché ... (Il Principe, Kap. IX: 80)

(Denn diese Fürsten üben die Gewalt entweder persönlich aus oder durch ihre Verwaltungen; im letzteren Fall haben sie einen schlechteren und gefährlicheren Stand, da ...)

(25) E come che il re conoscesse il luogo, là dove era, dovere esser tale che copiosamente di diverse salvaggine avervi dovesse, e l'avere davanti significata la sua venuta alla donna

\footnotetext{
${ }^{11}$ Renzi/Salvi: 478.
} 
spazio l'avesse dato di poter far cacciare, non pertanto [...] in altro non volle prender cagion di doverla mettere in parole se non delle sue galline; (Decameron, 1. Tag, 5. Novelle: 72) [Verweis auf das logische Infinitivsubjekt (il re) durch das Possessivpronomen: la sua venuta]

(Da der König wusste, dass es in der Gegend auch Wild in Fülle geben musste, und er seinerseits seine Ankunft rechtzeitig genug angekündigt hatte, so dass sie ihre Leute hätte auf die Jagd schicken können, wollte er ihr [...] gerade mit Hilfe dieser Hühner eine Falle stellen.)

Die in den zitierten Beispielsätzen eingebetteten Infinitivkonstruktionen sind durch die folgenden pragmatisch-kommunikativen Eigenschaften gekennzeichnet:

a) Thematizität (das heißt, sie stehen nicht im Mitteilungszentrum der jeweiligen Äußerung);

und

b) Präsupposition der existentiellen Gegebenheit des durch die Infinitivkonstruktion bezeichneten Sachverhalts.

Cfr. diesbezüglich noch einmal Beispiel (23):

l'avermi affaticato apresso di voi non poco mi gioverà, ...

Die Infinitivphrase ist durch einen Subjektsatz mit thematischem Konjunktiv substituierbar:

che mi sia affaticato apresso di voi non poco mi gioverà,...

Cfr.:

mi sono affaticato

$\uparrow$

Mitteilung eines Sachverhalts (Prädikation);

$\uparrow$

Indikativ als Kennzeichen des assertierend-mitteilenden

Charakters der Äußerung che mi sia affaticato

bzw.

l'avermi affaticato

\section{$\uparrow$}

Benennung eines Sachverhalts, dessen Gegebensein vorausgesetzt wird;

$\uparrow$

Konjunktiv und Infinitiv

als Kennzeichen/Modi der

Nicht-Mitteilung

Konstruktionen des Typs "Definiter Artikel + Infinitiv + Subjekt" machen die sogenannte "Doppelnatur" des Infinitivs deutlich, der formal und funktional zwischen Nomen und Verbum steht. Für die Nominalität der Konstruktion spricht die Verbindung des Infinitivs mit dem definiten Artikel, der prinzipiell die zwei folgenden pragmatisch-kommunikativen Eigenschaften hat:

- $\quad$ er löst (Existenz-)Präsuppositionen aus ("Voraussetzung der existentiellen Gegebenheit eines Sachverhalts" - s. o.) und fungiert somit generell als

- Kennzeichen thematischer Äußerungselemente ("Voraussetzung des informationellen Gegebenseins" - s. o.).

Der verbale Charakter des Infinitivs zeigt sich unter anderem an der expliziten Kodierung seines logischen Subjekts (cfr.: lo stare loro) sowie an den Formen des "infinito passato" (cfr.: l'avere davanti significata la sua venuta) und an den - durch die Valenz des Infinitivs geforderten - Ergänzungen (cfr.: l'avere davanti significata la sua venuta).

Salvi (1983: 243) unterscheidet generell zwischen "l'uso nominale e l'uso verbale dell'infinito preceduto da articolo" (nominaler und verbaler Gebrauch des durch einen Artikel eingeleite- 
ten Infinitivs). Als typische Eigenschaften des "infinito nominale" nennt er etwa dessen Verbindung mit einem nachgestellten Adjektiv ("aggettivo posposto") und/oder das Vorhandensein eines durch eine Präposition angeschlossenen Subjekts ("soggetto preposizionale") (cfr. Salvi 1983: 244-247):

\section{(26) l'avviarsi lento del treno}

(das langsame Anfahren des Zuges) [übers. v. Verf.]

Im Gegensatz dazu zählen beispielsweise Formen des "infinito passato" (s. o.), Anhebungskonstruktionen ("costruzioni a sollevamento", Raising), die Modifikation durch ein Adverb sowie das Vorhandensein klitischer Pronomina zu den verbalen Merkmalen des Infinitivs. Cfr.:

(27) L'aver Piero ricercato la verità (infinito passato)

(die Tatsache, dass Piero die Wahrheit gesucht hat)

(wörtlich: das haben Piero gesucht die Wahrheit) [übers. v. Verf.]

(28) Piero pare essere stanco (Anhebung/Raising) ${ }^{12}$

(Piero scheint müde zu sein)

(29) Il tuo credergli incondizionatamente (klitisches Pronomen, Adverb)

(die Tatsache, dass du ihm bedingungslos glaubst)

(wörtlich: dein glauben ihm bedingungslos) [übers. v. Verf.]

Was nun die verbale Verwendung der Konstruktion "Artikel + Infinitiv" betrifft, so können im Hinblick auf die unterschiedliche Kodierung des Infinitivsubjekts drei Typen differenziert werden:

Fra gli ess, di uso verbale dell'infinito articolato possiamo distinguere [...] tre tipi: quelli in cui è presente un soggetto lessicale [L'aver Piero ricercato la verità], quelli in cui, superficialmente, non abbiamo un soggetto [Il ricercare incessantemente la verità] e quelli in cui il soggetto è rappresentato da un pronome possessivo [Il tuo credergli incondizionatamente] [...]. (Salvi 1983: 250)

(Unter den Beispielen für den verbalen Gebrauch des durch einen Artikel eingeleiteten Infinitivs können drei Typen differenziert werden: jene, die ein lexikalisches Subjekt enthalten [L'aver Piero ricercato la verità]; jene, die kein oberflächensyntaktisches Subjekt aufweisen [Il ricercare incessantemente la verità], sowie jene, deren Subjekt in Form eines Possessivpronomens erscheint [Il tuo credergli incondizionatamente].) [übers. v. Verf.]

Der Konstruktionstyp in (27) (L'aver Piero ricercato la verità) stellt nach Salvi (1983: 251) die kanonische Form aller Sätze/Phrasen dar, die ein infinites Verbum und ein lexikalisches Subjekt aufweisen (cfr. Rizzi 1982, Kap. III).

Cfr. in diesem Zusammenhang das nächste Beispiel:

(30) * La causa del processo era un aver Piero rubato due milioni. (Salvi 1983: 252)

$$
[*+\text { rhematisch, }+ \text { faktiv }]
$$

(wörtlich: Die Ursache des Prozesses war ein haben Piero gestohlen zwei Millionen) [übers. v. Verf.]

Die Infinitivphrase (un aver Piero rubato due milioni) weist einen hohen Informationsgehalt auf, das heißt, sie stellt den rhematischen Teil der Äußerung dar, wobei die existentielle Gegebenheit des durch sie bezeichneten Sachverhalts vom Sprecher präsupponiert wird:

${ }^{12}$ Die Anhebungskonstruktion in (28) lässt sich aus dem folgenden zugrundeliegenden Satz ableiten: Pare che Piero sia stanco $\rightarrow$ Piero pare essere stanco. 
- Che cosa era la causa del processo?

(Was war die Ursache des Prozesses?)

- La causa del processo era che Piero aveva rubato due milioni.

(Die Ursache des Prozesses war, dass Piero zwei Millionen gestohlen hatte.)

Auch bei Substitution des indefiniten durch einen definiten Artikel (= Präsuppositionsauslöser) bleibt die Konstruktion inakzeptabel:

(31) * La causa del processo era l'aver Piero rubato due milioni.

$$
[*+\text { rhematisch, + faktiv] }
$$

In ihrer Funktion als Modus der Nicht-Mitteilung kann die Infinitivphrase des Typs "Artikel + Infinitiv + Subjekt" prinzipiell keine Äußerungsteile beinhalten, die sich auf ein Faktum beziehen und gleichzeitig rhematisch sind.

Cfr. dazu aber das folgende altitalienische Beispiel:

(32) e benché tra loro fussi qualche capo da fare alterazione, tamen dua cose li ha tenuti fermi: l'una, la grandezza della Chiesa, [...]; l'altra el non avere loro cardinali, li quali sono origine de' tumulti infra loro. (Il Principe, Kap. XII: 92)

(und wenn es auch unter ihnen etliche Aufwiegler geben mochte, so verhielten sie sich doch aus zwei Gründen ruhig: zum einen war es die Macht der Kirche, [...]; zum anderen hatten sie aus ihren Reihen keine Kardinäle, welche nämlich die Ursache für Unruhen unter ihnen bilden.)

Die durch einen bestimmten Artikel eingeleitete Infinitivphrase mit explizit genanntem Subjekt (el non avere loro cardinali) weist einen hohen Mitteilungswert auf. Dennoch fungiert auch in diesem Falle der Infinitiv als Modus der Nicht-Mitteilung, da der Sprecher den durch die Infinitivphrase kodierten Sachverhalt nicht behauptet, sondern dessen existentielle Gegebenheit präsupponiert. Im Allgemeinen sind Phrasen des Typs "Definiter Artikel + Infinitiv + Subjekt" jedoch durch Thematizität bzw. durch einen geringen Mitteilungswert gekennzeichnet (cf. noch einmal Bsp. 22-25).

$\mathrm{Da}$ thematische Elemente generell Richtung Satzanfang tendieren, steht auch die (thematische) Infinitivphrase im Allgemeinen vor dem Prädikat (cf. Salvi 1983: 252).

Also:

(33) L'aver Giorgio invitato quella donna è vergognoso. (Salvi 1983: 266)

[+ thematisch, + faktiv]

(Dass Giorgio diese Frau eingeladen hat, ist unverschämt.) [übers. v. Verf.]

aber nicht:

(34) * È vergognoso l'aver Giorgio invitato quella donna.

(Es ist unverschämt, dass Giorgio diese Frau eingeladen hat.)

(35) *Non sopporto l'essere Giorgio così smemorato. (ebd.)

(Ich ertrage nicht, dass Giorgio so vergesslich ist.) [übers. v. Verf.]

Man betrachte diesbezüglich auch die zwei nächsten Beispielsätze (Salvi 1983: 249, 252):

(36) Conoscere la famiglia di lui è dunque un integrar la conoscenza della sua persona.

$$
\text { [+ rhematisch, - faktiv] }
$$

(Seine Familie kennen zu lernen bedeutet folglich, die Kenntnis seiner Person zu vervollständigen.) [übers. v. Verf.] 
(37) Quello che si temeva era un suo eventuale farsi frate.

$$
\text { [+ rhematisch, - faktiv] }
$$

(Was man fürchtete war, dass er Mönch werden könnte.) [übers. v. Verf.]

In beiden Fällen stellt die durch den indefiniten Artikel eingeleitete Infinitivphrase den rhematischen Äußerungsteil dar. Der Unterschied zu (30) besteht jedoch darin, dass die Infinitivphrase kein explizit genanntes nachgestelltes Subjekt enthält und dass der Sprecher die Gegebenheit des durch die Infinitivphrase bezeichneten Sachverhalts nicht präsupponiert. Cfr. dazu Salvi (1983: 252):

[...] se non c'è soggetto [...] o se il soggetto è rappresentato da un pronome possessivo [...], possiamo avere l'articolo indeterminativo, $[\ldots]$

(wenn kein Subjekt vorhanden ist [...] oder wenn das Subjekt durch ein Possessivpronomen repräsentiert wird [...], kann der unbestimmte Artikel stehen) [übers. v. Verf.]

Cfr. dazu auch die folgenden altitalienischen Beispiele, die Ähnlichkeiten mit dem bisher beschriebenen Konstruktionstyp (Definiter Artikel + Infinitiv + Subjekt) aufweisen: Durch die Einbettung in eine Infinitivphrase werden bestimmte Äußerungsteile als nicht-assertierend und folglich als thematisch gekennzeichnet. Die Infinitivphrase selbst ist von einem Ausdruck der subjektiven Bewertung abhängig, der das Mitteilungszentrum (Rhema) der Äußerung darstellt. Der Infinitiv fungiert somit als Modus der Nicht-Mitteilung, wobei das (unpersönliche) Infinitivsubjekt explizit kodiert und dem Infinitiv nachgestellt wird ${ }^{13}$ :

(38) a Dio uccidere sé stesso l'omo è peccato che ... (Guittone)

(für Gott ist sich selbst zu töten eine Sünde, die ...)[übers. v. Verf.]

(39) stare l'uomo ozioso e non operare fa contro natura (Giordano da Rivalto)

(Müßiggang und Tatenlosigkeit sind gegen die Natur des Menschen.) [übers. v. Verf.]

(40) esser costante l'om è cosa altera (Bonichi)

(Beständigkeit ist ein hohes Gut.) [übers. v. Verf.]

(41) è una grande fatica a conservare l'uomo la pecunia (Giordano da Rivalto)

(Es kostet viel Mühe, sein Geld zusammenzuhalten.) [übers. v. Verf.]

Nach Segre (1991: 118) können Infinitivphrasen mit einem explizit kodierten unpersönlichen Subjekt (иото) nicht als Subjektsätze im eigentlichen Sinne verstanden werden:

[...] non sono delle vere proposizioni soggettive: sono semplici infiniti a cui fu aggiunto un soggetto impersonale (l'uomo). E ciò risponde a una tendenza del Due e Trecento [...].

(es handelt sich nicht um Subjektsätze im eigentlichen Sinn, sondern um einfache Infinitivformen, denen ein unpersönliches Subjekt hinzugefügt wurde (l'uomo). Das entspricht einer Tendenz des dreizehnten und vierzehnten Jahrhunderts ...) [übers. v. Verf.]

\section{Schlussbemerkung mit einigen Anmerkungen zum Grundwert des Konjunktivs}

Die vorliegende Arbeit hat gezeigt, dass die Infinitivkonstruktion des Typs "Definiter Artikel + Infinitiv + Subjekt" pragmatisch-kommunikative Gemeinsamkeiten mit dem thematischen Konjunktiv aufweist. In den zitierten Beispielen wurden einzelne Äußerungsteile durch ihre Einbettung in eine Infinitivphrase als nicht-assertierend (cfr. Assertion vs. Präsupposition) gekennzeichnet. In diesem Sinne fungiert der Infinitiv (mit Artikel) gewissermaßen als Modus der Nicht-Mitteilung. Diese syntaktisch-pragmatische Funktion des Infinitivs wird gerade an seiner sogenannten "Doppelnatur" offensichtlich, steht er doch sowohl formal als auch

${ }^{13}$ Zitiert bei: Segre, Lingua, stile e società. Studi sulla storia della prosa italiana: 117-119. 
funktional zwischen Nomen und Verbum. Als nominale Eigenschaft wurde neben dem Merkmal [- finit] beispielsweise die Verbindung mit einem Artikel genannt. Zu den verbalen Charakteristika zählen u. a. die im Altitalienischen übliche explizite Kodierung des Infinitivsubjekts sowie das Vorhandensein von Infinitivergänzungen (Komplementen). Cf. Gsell/Wandruszka (1986: 2):

Jeder Satz mit einer finiten Verbform muss, sofern er als selbständige Äußerung auftritt, in irgendeiner Weise [...] Stellung nehmen zur Geltung, zum Außenweltbezug seines Inhaltes. Nicht-finite Verbformen hingegen [...] enthalten keinen Hinweis auf einen solchen Bezug; sie nennen nur, ohne etwas zu sagen (zu prädizieren).

Diese Funktion, "etwas zu nennen, ohne zu prädizieren", erfüllt in vergleichbarer Weise auch der thematische Konjunktiv. Als Kennzeichen thematischer Äußerungselemente versprachlicht er gerade jene Inhalte, die nicht das Mitteilungsziel - also das, was der Sprecher primär kommunizieren möchte - darstellen (= Modus der Nicht-Mitteilung; z. B.: Je suis content [Rhema] que tu sois venu [Thema/thematischer Konjunktiv]).

Was den Konjunktiv im Allgemeinen (nicht nur den thematischen Konjunktiv) betrifft, so hat die Suche nach einem möglichen (übereinzelsprachlichen) Grundwert unterschiedlichste Definitionen hervorgebracht, wobei "bislang jedoch alle Versuche, diesen Grundwert genau zu bestimmen und allgemein akzeptiert zu formulieren, gescheitert sind" (Hummel 2001: 49). Cf.:

In Sätzen wie Lamento que esté enfermo [Je regrette qu'il soit malade] liegt Tatsächlichkeit vor. Das Ereignis ist nicht vorgestellt, sondern real eingetreten. Interessanter ist die Beobachtung [...], wonach der Subjunktivgebrauch [...] nicht von der Tatsächlichkeit des Ereignisses abhängt, d. h. es kann eingetreten sein oder auch nicht. [...] Mit dem Subjunktiv abstrahiert der Sprecher von der Tatsächlichkeit. Dies kann er gedanklich auch in den Fällen, in denen Tatsächlichkeit vorliegt [...] (Hummel 2001: 26)

Die eigentliche Frage ist daher nicht, ob ein Sachverhalt tatsächlich eingetreten ist oder nicht, sondern vielmehr "wie der Subjunktiv diesen Sachverhalt im Unterschied zum Indikativ präsentiert" (Hummel 2001: 96). Cf. dazu Kalepky (1928: 71):

Denn wenn ich zu jemand sage: Je suis content que tu sois venu, so [...] abstrahiere ich beim Ausdruck meiner Freude von dieser Tatsache, betone nicht ihre Realität, sondern deute sie nur als Grund meiner Freude an, stelle sie also nicht als wahrgenommen, sondern nur als in meinem Geiste vorhanden dar.

Die Auffassung, ein möglicher Grundwert des Subjunktivs bestehe in seiner Funktion als Modus der Nicht-Mitteilung, ist freilich nicht neu und kann auf einen bereits von Kalepky und Lerch (et al.) ausgearbeiteten "psychologischen Ansatz" zurückgeführt werden. ${ }^{14}$ Lerch spricht nicht von thematischem Konjunktiv, sondern verwendet stattdessen den Ausdruck Konjunktiv des psychologischen Subjekts (unter psychologischem Subjekt sind die dem Hörer bereits bekannten, thematischen Äußerungselemente zu verstehen) (cfr. Lerch 1920: 339). Auch die von Gsell/Wandruszka (1986: 21) vorgeschlagene negative Bestimmung des Grundwertes basiert auf syntaktisch-pragmatischen Kriterien:

Der Subjonctif und allgemein der romanische Konjunktiv tritt nicht in Sätzen auf, die ein Faktum wiedergeben und zugleich rhematisch sind (als Mitteilungsziel fungieren).

Cf. Hummel (2001: 46):

Parallel zu dieser diskursiv-pragmatischen Neuausrichtung der Grundwertdiskussion ist die [...] an Äußerungsmerkmalen wie "Affirmation", "Nicht-Affirmation" und "Präsupposition"

${ }^{14}$ Cf. Hummel, Der Grundwert des spanischen Subjunktivs, Kap. "Forschungsüberblick zum Grundwert des Subjunktivs": 31-33. 
orientierte Definition des Grundwerts von Indikativ und Subjunktiv zu nennen. Sie bildet den Ausgangspunkt für eine neue Analyserichtung, die vor allem von Forschern in den USA und Hispanoamerika, jüngst aber auch in Spanien, vertreten wird. Zentraler Ansatzpunkt ist die Annahme, dass die Modi sich auf die Aussage beziehen, also nicht auf die bezeichneten außersprachlichen Ereignisse [...]. Terrell/Hooper [...] schlagen [...] eine semantische Bestimmung des Grundwerts vor, wonach der Indikativ sich durch das Merkmal [+ Affirmation] und der Subjunktiv durch das Merkmal [- Affirmation] auszeichne:

We can thus hypothesize that in independent and noun clauses the indicative is associated with assertion while the subjunctive is associated with non-assertion. (Terrell/Hooper 1974: 487)

Die Unterscheidung zwischen assertorischen und nicht-assertorischen Äußerungen findet sich auch bei Lavandera. Cf. dazu Palmer (1986: 17):

In a similar way Lavandera (1983: 211), with specific reference to the subjunctive in Spanish, distinguishes [+ assertive] and [-assertive] utterances, and says that utterances in the subjunctive "do not refer to states or events whose occurrence is questionable, or just feared, wished, doubted, etc., but to "states of affairs" whose occurrence could easily be denied or affirmed, but is instead left unasserted".

\section{$8 \quad$ Fazit}

Eine wesentliche gemeinsame kommunikationsspezifische Funktion von Infinitiv und (thematischem) Konjunktiv besteht darin, Äußerungsteile als Nicht-Mitteilung und folglich als thematisch zu kennzeichnen.

Parallel zum thematischen Konjunktiv kann man daher auch von einem thematischen Infinitiv sprechen:

De même qu'une complétive en antéposition, l'infinitif présente un thème par rapport auquel la proposition qui suit prend position. (Togeby 1983: 74)

(Ebenso wie ein vorangestellter Komplementsatz repräsentiert der Infinitiv ein Thema, zu dem die darauffolgende Proposition Stellung nimmt.) [übers. v. Verf.]

Der Infinitiv, das heißt die Infinitivphrase des Typs "Definiter Artikel + Infinitiv + Subjekt", stellt - ebenso wie der thematische Konjunktiv - ein morphosyntaktisches Mittel zur Thematisierung eines Satzes bzw. eines bestimmten Äußerungsteiles dar.

\section{Primärliteratur}

Alberti, Leon Battista, I libri della famiglia, a cura di Ruggiero Romano, Torino: Einaudi, 1972, 2. ed. (Nuova universale Einaudi; 102).

Alberti, Leon Battista, Über das Hauswesen, übersetzt von Walther Kraus, Zürich u.a.: Artemis-Verlag, 1962 (Die Bibliothek der Alten Welt: Reihe Antike und Humanismus).

Boccaccio, Giovanni, Decameron, übersetzt und herausgegeben von Peter Brockmeier, Stuttgart: Philipp Reclam jun., 1988.

Machiavelli, Niccolò, Il Principe, übersetzt und herausgegeben von Philipp Rippel, Stuttgart: Philipp Reclam jun., 1986.

Speroni, Sperone, Dialogo delle lingue, herausgegeben, übersetzt und eingeleitet von Helene Harth, München: Wilhelm Fink Verlag, 1975.

\section{Sekundärliteratur}

Blumenthal, Peter (1987): Sprachvergleich Deutsch - Französisch. Tübingen.

Franks, Steven (1995): Parameters of Slavic Morphosyntax. New York etc.

Gsell, Otto/Wandruszka, Ulrich (1986): Der romanische Konjunktiv. Tübingen. 
Halliday, Michael A. K. (1985): An Introduction to Functional Grammar. London.

Hummel, Martin (2001): Der Grundwert des spanischen Subjunktivs. Tübingen.

Jansen, Hanne/Polito, Paola/Strudsholm, Erling (2002): "Dialogo vago sull'infinito e altro." In: Jansen, Hanne et al. (eds.) (2002): L'infinito \& oltre. Omaggio a Gunver Skytte. Odense: 9-28.

Kalepky, Theodor (1928): "Verwechslung von Grundbedeutung und Gebrauchsweise in der französischen Tempus- und Moduslehre." Zeitschrift für Romanische Philologie 48: 5374.

Lavandera, Beatriz R. (1983): "Shifting moods in Spanish discourse." In: Klein-Andrew, Flora (ed.) (1983): Discourse perspectives on syntax. New York: 209-236.

Lerch, Eugen (1920): "Der Konjunktiv des psychologischen Subjekts im Französischen." Die neueren Sprachen 27: 338-344.

Mayerthaler, Willi/Fliedl, Günther/Winkler, Christian (1993): Infinitivprominenz in europäischen Sprachen. Teil I: Die Romania (samt Baskisch). Tübingen.

Maurice, Florence (1996): Der modale Infinitiv in der modernen russischen Standardsprache. München.

Palmer, Frank Robert (1986): Mood and Modality. Cambridge.

Ramat, Paolo (2002): "La natura dell'infinito." In: Jansen, Hanne et al. (eds.): L'infinito \& oltre. Omaggio a Gunver Skytte. Odense: 409-417.

Renzi, Lorenzo/Salvi, Giampaolo (eds.) (1991): Grande grammatica italiana di consultazione. Volume II, I sintagmi verbale, aggettivale, avverbiale. La subordinazione. Bologna.

Reumuth, Wolfgang/Winkelmann, Otto (1993): Praktische Grammatik der italienischen Sprache. 4. Auflage, Wilhelmsfeld.

Rizzi, Luigi (1982): Issues in Italian Syntax. Dordrecht.

Salvi, Giampaolo (1983): "L'infinito con l'articolo." In: Franchi De Bellis, Annalisa/Savoia, Leonardo M. (eds.) (1985): Sintassi e morfologia della lingua italiana d'uso. Teorie e applicazioni descrittive. Atti del XVII congresso internazionale di studi, Urbino. Rom: 243-268. (= Pubblicazioni della Società di Linguistica Italiana 24)

Segre, Cesare (1991): Lingua, stile e società. Studi sulla storia della prosa italiana. Milano.

Skytte, Gunver (1983): La sintassi dell'infinito in italiano moderno. Vol. I-II. Kopenhagen.

Terrell, Tracy/Hooper, Joan (1974): "A Semantically Based Analysis of Mood in Spanish." Hispania 57: 484-494.

Togeby, Knud (1983): Grammaire française. Volume III: Les Formes Impersonnelles du Verbe et la construction des verbes. Études Romanes de l'Université de Copenhague.

Tschida, Alexander (1995): Kontinuität und Progression. Entwurf einer Typologie sprachlicher Information am Beispiel des Französischen. Wilhelmsfeld.

Vallauri Lombardi, Edoardo (1996): La sintassi dell'informazione. Uno studio sulle frasi complesse tra latino e italiano. Roma.

Wandruszka, Ulrich: "Nochmals zum 'thematischen' Konjunktiv." In: Heinz, Sieglinde/ Wandruszka, Ulrich (eds.) (1982): Fakten und Theorien. Festschrift für Helmut Stimm. Tübingen: 343-351.

Winkler, Christian (1993): Modus und Modalität im Italienischen am Beispiel von Infinitiv und Konjunktiv. Diplom-Arbeit, Universität Klagenfurt.

Wunderli, Peter (1976): Modus und Tempus. Beiträge zur synchronischen und diachronischen Morphosyntax der romanischen Sprachen. Tübingen. 